\title{
Evaluation of the level of awareness of congenital toxoplasmosis and associated practices among pregnant women and health workers in Tanzania's Temeke district in Dar es Salaam
}

\author{
Onduru Gervas Onduru¹, Susan Fred Rumisha², Musso Munyeme ${ }^{1}$, Andrew Malata Phiri ${ }^{3}$
}

1. University of Zambia School of Veterinary Medicine, Disease Control.

2. National Institute for Medical Research, Directorate of Information Technologies and Communication.

3.University of Zambia School of Veterinary Medicine, clinical studies.

\begin{abstract}
Background: Toxoplasmosis caused by the obligate intracellular coccidian protozoan Toxoplasma gondii (T. gondii) infects all warm-blooded animals including humans. This parasite may develop in both immune-compromised and immunocompetent hosts but usually the disease manifestations strongly differ according to immune status. Immunocompromised hosts develop more severe disease than immunocompetent hosts. Infections in pregnancy carry the risk of foetal involvement and can lead to serious clinical outcomes including psychomotor and ocular disorders in congenitally infected foetuses and children.

Objective: To assess the level of awareness and practices towards congenital toxoplasmosis among health workers and pregnant women in Tanzania's Temeke municipality.

Methods: This was a cross-sectional study involving 371 pregnant women and 22 health workers from six healthcare facilities in Temeke municipality of Dar es Salaam, Tanzania. A structured questionnaire and review of prenatal screening forms were used to collect information. The questionnaire focused on knowledge of disease aetiology, signs and symptoms, modes of transmission, treatment and management.

Results: Of the pregnant women, 96\% (95\% CI: 0. 94-0.98) were unaware of the disease, had never heard, read or seen any information regarding toxoplasmosis. The majority of respondents including those who had heard, read or seen information concerning toxoplasmosis were unaware of the disease aetiology, signs and symptoms. However, 90\% (95\% CI: 0.86-0.93) of respondents unknowingly observed preventive practices towards the disease including avoiding eating raw, cured or rare meat. There was a significant statistical relationship between practices towards toxoplasmosis and age of pregnant women, such that for every increase in age by ten years the risk practices towards toxoplasmosis increased by $41 \%$ (OR=1.41, 95\%, C.I. 1.05-1.90). Preventive practices towards toxoplasmosis decreased significantly by $74 \%$ and $78 \%$ for the age of $19-25$ and $26-35$ years old pregnant women respectively, as compared to those $<19$ years. No significant difference was observed for those aged $>35$ years. Multigravidae was associated with at-risk practices towards toxoplasmosis (OR=2.65, CI: 1.38-5.08). Of the 22 health workers who participated in the study, 36\% (95\% CI: 0.15-0.58) were aware of the congenital toxoplasmosis and its clinical outcomes. None of them had diagnosed the disease before.

Conclusion: Due to general lack of awareness towards toxoplasmosis observed among both health workers and pregnant women in Temeke Municipality, we recommend health policy on maternal and child healthcare to address prenatal screening that is aimed at providing early diagnosis for any possible congenital toxoplasmosis as well as diseases that are currently screened in Tanzania such as HIV, syphilis and malaria. Integrating a One Health approach in educating medical professionals and the vulnerable population of pregnant women on the importance of congenital zoonoses will promote awareness and preventive practices towards the disease.
\end{abstract}

Keywords: Toxoplasmosis awareness, pregnant women, health workers, Dar es Salaam, Tanzania.

DOI: https://dx.doi.org/10.4314/ahs.v19i4.24

Cite as: Onduru OG, Rumisha SF, Munyeme M, Phiri AM. Evaluation of the level of awareness of congenital toxoplasmosis and associated practices among pregnant women and health workers in Tanzania's Temeke district in Dar es Salaam. Afri Health Sci.2019;19(4):3027-3037. https:// dx.doi.org/10.4314/abs.v19i4.24

\section{Corresponding author:}

Onduru Gervas Onduru,

University of Zambia School of Veterinary Medicine,

Disease Control.

Email: ogyonduru@yahoo.com

\section{Introduction}

Toxoplasmosis is a zoonotic disease caused by the obligate intracellular coccidia protozoan, Toxoplasma gondii. The parasite belongs to apicomplexa family in the genus Toxoplasma and it is the only known species of the genus Toxoplasma ${ }^{1}$. All warm-blooded animals including
(C) 2019 Onduru et al. Licensee African Health Sciences. This is an Open Access article distributed under the terms of the Creative commons Attribution License (https://creativecommons.org/licenses/BY/4.0), which permits unrestricted use, distribution, and reproduction in any medium, provided the original work is properly cited.
African Health Sciences Vol 19 Issue 4, December, 2019 
humans can function as intermediate hosts for $T$. gondii while felids are the only definitive hosts ${ }^{2}$. The majority of human infections in immunocompetent hosts are asymptomatic, minority may express mild "flu like" illness that is self-limiting. However, the disease may become fatal in immuneocompromised hosts resulting mostly in Toxoplasma encephalitis (TE) ${ }^{3}$.

The two main routes of Toxoplasma transmission are oral and congenital ${ }^{4}$. The most important sources of Toxoplasma infection are the resistant tissue cysts present in undercooked or raw meat and the oocysts found in faeces of infected cats ${ }^{5}$. Definite hosts are mainly infected through carnivorism i.e. eating small rodents. In humans, T. gondii infection can occur congenitally across the placenta ${ }^{6}$ and after birth through (i) ingestion of the parasite cyst in food such as raw or undercooked meat, or (ii) untreated drinking water, fruits or soil contaminated with cat faeces containing Toxoplasma oocyst. Other means of T. gondii transmission include organ transplant and blood transfusion ${ }^{6}$.

Therefore, this study was conducted to assess the awareness and practices towards congenital toxoplasmosis among health workers in Temeke and pregnant women attending antenatal care services at health facilities in Temeke municipality, Dar es Salaam, Tanzania.

Toxoplasmosis is still a neglected disease and under-reported in many parts of the world including Tanzania despite having a disease burden similar to salmonellosis and campylobacteriosis ${ }^{7}$. Routine prenatal screening services for congenital toxoplasmosis are still lacking in many developing countries. Primary infection of pregnant women during the third trimester entails greater risk of vrtical transmission of toxoplasmosis than earlier in pregnancy, but outcomes of infection are more severe when transmission occurs at the early stages of gestation ${ }^{8}$. Congenital infection and manifestations of toxoplasmosis in foetus and newborn are responsible for central nervous system problems and ocular disease ${ }^{9}$. These include retinitis, mental retardation, blindness, hydrocephalus, hemiparesis, encephalitis, seizure, disequilibrium, intracranial calcification and death'.

Toxoplasma gondii infection in humans is prevalent worldwide; the estimated rate of chronic $T$. gondii infection is $30-50 \%{ }^{10}$. Depending on the geographical settings and living conditions, the worldwide prevalence range from
$1-100 \%{ }^{11}$. In Africa, the highest prevalence of human toxoplasmosis was reported in western and Eastern Africa and the lowest prevalence in Southern African countries. Ghana recorded the highest prevalence of $92.5 \%{ }^{12}$, Nigeria, $75 \%{ }^{13}$, Benin, $30 \% 0^{14}$, Ethiopia, $85.4 \%{ }^{15}$, Madagascar, $80 \%{ }^{16}$, Uganda, $54 \%{ }^{17}$, Zambia, $5.87 \%{ }^{18}$, South Africa, $9.8 \%{ }^{19}$ and $44.6 \%$ in Swaziland ${ }^{20}$. In central and northern Africa, the prevalence was found to be high in the Democratic Republic of Congo (DRC) $80.3 \%{ }^{21}$ and Tunisia, $58.4 \% \%^{22}$.

In Tanzania, the prevalence of toxoplasmosis ranges from $4 \%$ to $60 \%$ depending on, geographical settings, age and occupation $^{23-26}$. A study by Swai and Schooman reported higher prevalence of toxoplasmosis in the adult population as compared to young age 26. In Mwanza region of Tanzania, 39\% of pregnant women were infected with Toxoplasma $a^{25}$ and $41.7 \%$ prevalence of chronic T. gondii infection was reported in pregnant women in Kilimanjaro (North-eastern part of Tanzania) $^{24}$. Previously, 4\% human toxoplasmosis prevalence was reported in Nyamisati village in Tanzania's Coastal region ${ }^{23}$. On the other hand, serological survey in animals also showed that $14.2 \%$ of goats and sheep were $T$. gondii seropositive in southern Tanzania ${ }^{23}$.

Despite the evidence of the burden of toxoplasmosis in Tanzania, there is a lack of information regarding the awareness and practices of pregnant women and health workers towards congenital toxoplasmosis. Previous studies on toxoplasmosis in the country were based on the determination of seroprevalence of the disease in adults. Therefore, the burden of congenital toxoplasmosis in newborns in Tanzania is missed. This knowledge gap limits the risk estimation for the occurrence of congenital toxoplasmosis and the availability of the data for the clinical presentation of toxoplasmosis in newborns born to HIV-negative and HIV-positive women. Similarly, the lack of information about pregnant women and healthcare workers awareness and practices towards toxoplasmosis also undermines and limits the scope of health care for pregnant women with regards to congenital infections.

Therefore, this study was conducted to assess the awareness and practices towards congenital toxoplasmosis among health workers in Temeke and pregnant women attending antenatal care services at health facilities in Temeke municipality, Dar es Salaam, Tanzania 


\section{Materials and methods}

\section{Study design and location}

A cross-sectional study was conducted between January and May, 2016, in six health facilities that had Reproduction and Child Health $(\mathrm{RCH})$ clinics in Temeke munici- pality, Dar es Salaam, Tanzania. The healthcare facilities included Rangitatu hospital, Roundtable maternity home, Tabukareli dispensary, Kibugumo dispensary, Vijibweni hospital and Kigamboni health centre as shown in Figure 1.

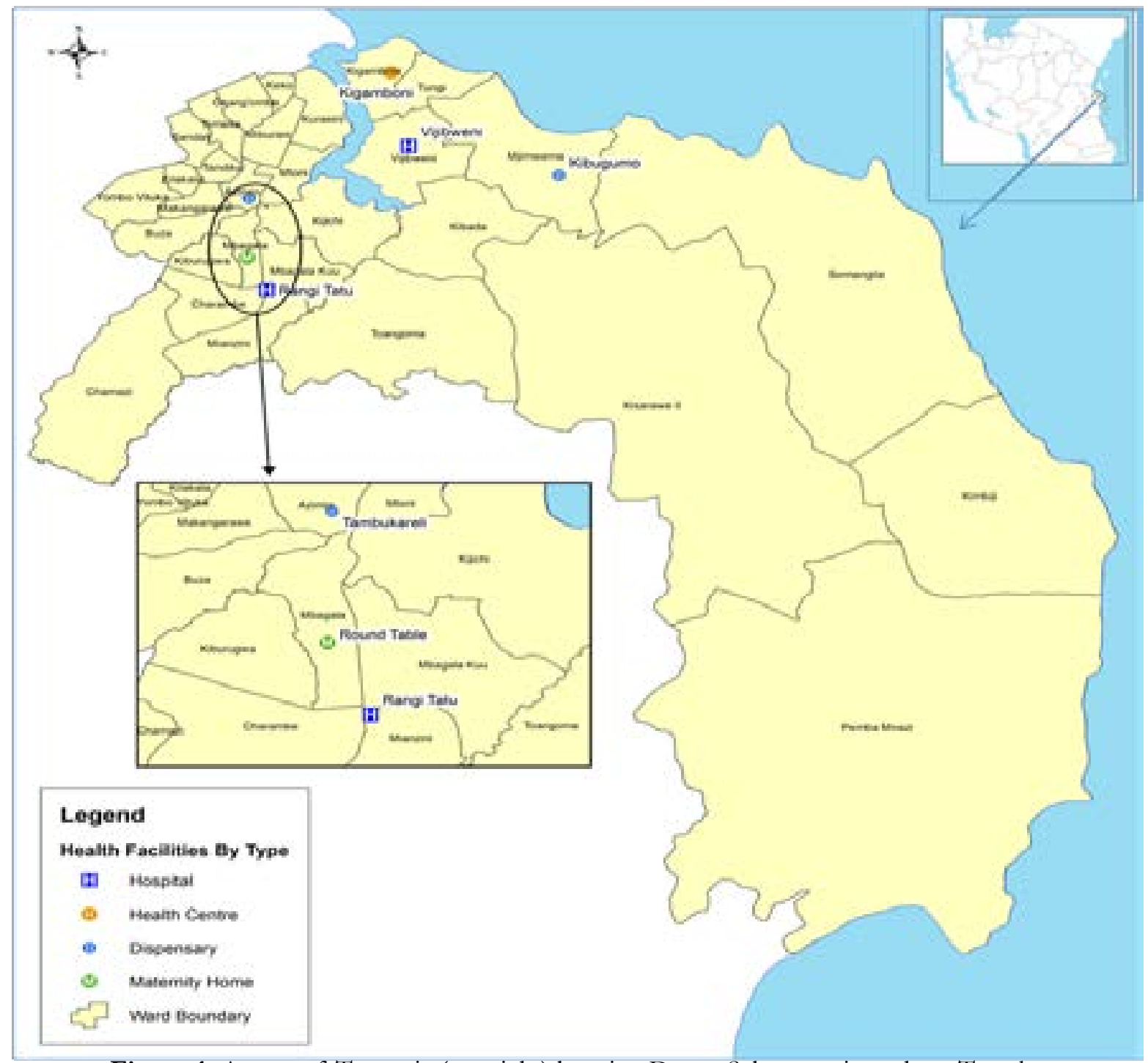

Figure 1: A map of Tanzania (top right) locating Dar es Salaam region where Temeke municipality belongs. From the map of Temeke municipality (insert left bottom), the wards and hospital locations of study sites are shown as indicated in the legend table.

\section{Sampling and sample size}

This study involved 22 health workers providing health care services in the RCH clinics and 371 pregnant women attending the $\mathrm{RCH}$ clinics for prenatal health services (393 total participants). The WHO formula for Sample Size Determination in Health Studies was used for sample size calculation 27. Briefly; $\mathrm{N}=(\mathrm{Z} 2 * \mathrm{P} *(1-\mathrm{P}) * \mathrm{D}) / \mathrm{E} 2$ $\mathrm{N}=$ number of respondents (sample population). $\mathrm{P}=$ Expected knowledge level of the disease among pregnant women in Tanzania. Since no information was found on this, a conservative $\mathrm{P}=0.5$ was assumed.
$\mathrm{Z}$ is a $\mathrm{Z}$-value corresponding to the $5 \%$ level of significance.

$\mathrm{D}$ is a design effect which was set as 1

$\mathrm{E}$ is a margin of error (precision), taken as 0.05 .

Therefore the minimum sample size of 384 was obtained as follows;

$\mathrm{N}=(1.962 * 0.5(0.5) * 1) /(0.05) 2=\mathrm{N} \sim 384$.

During the study, the actual number of participants surveyed became 393 which was proportionally shared between two categories of study participants. The sample proportion of participants included $94 \%$ pregnant women and 5.6\% healthcare workers. 
Based on the population distribution and geographical location within urban and peri-urban settings of Temeke municipality, stratified sampling was used to select three wards from an urban area and two wards from peri-urban area. From each ward, at least one RCH clinic which serves many pregnant women was purposively chosen. There were no special criteria for inclusion of the pregnant women; all who attended a clinic on the day of survey had the same chance of being included. A convenience sample of pregnant women was taken whereas health workers who attended to pregnant women and were present on the day of data collection were included in the study. The questionnaire was administered by the interviewer and all responses were recorded verbatim.

\section{Data collection}

Data for this study were collected through a questionnaire and observational review of prenatal screening forms or antenatal clinic cards. There were two sets of pretested questionnaires developed for each group of participants, one for pregnant women and the other for health workers. Closed and open structured questionnaires were used. Questions focused on knowledge of the disease aetiology, signs and symptoms, modes of transmissions, treatment and management. Population characteristics of pregnant women included age, economic activity, education level, marital status, gestational age, abortion history and gravidity.

\section{Data analysis}

Descriptive statistics and logistic regression analysis were computed using STATA version 12 (Stata Corp LP, College Station, USA). Population characteristics were described as counts and percentages. Awareness towards toxoplasmosis was described according to the participant's responses to the questions. The association between demographic data and risk practices of the respondents towards toxoplasmosis was measured using chi-squared test and logistic regression model (bivariate and multivariate).

\section{Ethical considerations}

Ethical clearance to conduct the study was granted by the National Institute for Medical Research in Tanzania (NIMR/HQ/R.8a/Vol. IX/2127). The permission from Temeke Municipal Research Committee was granted for the research to be done in the health facilities under Temeke Municipal Council. Voluntary participation was sought from each participant and confirmed by signed informed consent form.

\section{Results \\ Descriptive characteristics}

A total of 22 health workers and 371 pregnant women from six health facilities in Temeke Municipality participated in the study. The distribution of pregnant women and health workers varied from one health centre to another. The highest percentage of pregnant women interviewed were from Roundtable maternity home in an urban area $(23.9 \%, \mathrm{n}=89)$, the least representation of pregnant women was from Kibugumo dispensary in a peri-urban area $(2.4 \%, \mathrm{n}=9)$. From Rangitatu hospital $20.8 \%(n=77)$ pregnant women were sampled, Tabukareli dispensary $21.7 \%(\mathrm{n}=80)$, Vijibweni hospital $15.3 \%$ $(n=57)$ and $15.9 \%(n=59)$ were from Kigamboni health centre.

The ages of pregnant women ranged from 12 to 52 years old and the mean age was 26.9 years. Participants younger than 19 years accounted for $11 \%$. The largest age group was those in years between 19 and 25 (42\%). Thirty-three per cent of pregnant women were in the age between 2635 years and those above 35 years were 14\%.

\section{Awareness level of pregnant women regarding toxo- plasmosis}

Amongst 371 pregnant women surveyed, 355 (95.7\%) 95\% CI: 93.6-97.7\% were unaware that there was a disease called toxoplasmosis; i.e. they had never heard, read or seen any information regarding toxoplasmosis. Pregnant women who had heard, read or seen information concerning toxoplasmosis $4.3 \%(\mathrm{n}=16)$, (95\% CI: 2.2 $6.3 \%)$ were generally unaware of the aetiology, signs and symptoms, modes of transmission, treatment and management of the disease. However, they had a partial understanding of the clinical manifestation of toxoplasmosis; 2 out of 16 who were aware (12.5\%) 95\% CI: $6-31 \%$ said it is associated with encephalitis and 6 pregnant women amongst 16 who had the knowledge (37.5\%) 95\% CI: $10-64 \%$ said the disease causes retinitis and mental problems such as hydrocephalus, microcephalus and mental retardation in children.

Of the 16 pregnant women who were aware of Toxoplasmosis, 13 (81.3\%) 95\% CI: 76.5-84.8\% were multigravidaee and showed high level of awareness compared to primigravidae. It was found that none of those who did not attend school had heard, seen, or read about toxoplasmosis (Table 1). 
Table 1: The characteristics of the pregnant women in the toxoplasmosis awareness study, Temeke, Dar es Salaam, Tanzania $(\mathrm{N}=371)$

\begin{tabular}{|c|c|c|c|}
\hline Factors & $\mathbf{n}$ & $\begin{array}{c}\text { Aware of } \\
\text { toxoplasmosis } \\
\text { n(\%) }\end{array}$ & $\begin{array}{c}\text { Unaware of } \\
\text { toxoplasmosis } \\
n(\%)\end{array}$ \\
\hline \multicolumn{4}{|l|}{ Age } \\
\hline$<19$ & 39 & $1(2.6 \%)$ & $38(97.4 \%)$ \\
\hline $19-25$ & 156 & $8(5.1 \%)$ & $148(94.9 \%)$ \\
\hline $26-35$ & 124 & $3(2.4 \%)$ & $121(97.6 \%)$ \\
\hline$>35$ & 52 & $4(7.7 \%)$ & $48(92.3 \%)$ \\
\hline \multicolumn{4}{|l|}{ Marital status } \\
\hline Single & 51 & $1(2 \%)$ & $50(98 \%)$ \\
\hline Married & 295 & $12(4.1 \%)$ & $283(95.9 \%)$ \\
\hline Separated/divorced & 25 & $3(22 \%)$ & $22(88 \%)$ \\
\hline \multicolumn{4}{|l|}{ Education } \\
\hline Did not attend school & 26 & $0(0 \%)$ & $26(100 \%)$ \\
\hline Primary & 236 & $9(3.8 \%)$ & $227(96.2 \%)$ \\
\hline Secondary & 94 & $5(5.3 \%)$ & $89(94.7 \%)$ \\
\hline College/University & 15 & $2(13.3 \%)$ & $13(86.7 \%)$ \\
\hline \multicolumn{4}{|l|}{ Gestation age } \\
\hline 1st trimester & 51 & $1(2 \%)$ & $50(98 \%)$ \\
\hline 2nd trimester & 146 & $5(3.4 \%)$ & $141(96.6 \%)$ \\
\hline 3rd trimester & 174 & $10(5.7 \%)$ & $164(94.3 \%)$ \\
\hline \multicolumn{4}{|l|}{ Gravidity } \\
\hline Primigravidae & 123 & $3(2.4 \%)$ & $120(97.6 \%)$ \\
\hline Multigravidae & 248 & $13(5.2 \%)$ & $235(94.8 \%)$ \\
\hline \multicolumn{4}{|l|}{ Abortion History } \\
\hline Yes & 87 & $5(5.7 \%)$ & $82(94.3 \%)$ \\
\hline No & 284 & $11(3.9 \%)$ & $271(96.1 \%)$ \\
\hline
\end{tabular}

Practices of pregnant women towards toxoplasmosis Twenty six (7\%) 95\% CI: 4.3-9.6\% pregnant women kept cats and $20(5 \%) 95 \% \mathrm{CI}: 3.1-7.6 \%$ cleaned and changed cat litter. It was found that 333 (90\%) 95\% CI: 86.6$92.8 \%$ did not eat cured, rare and or raw meat. Similarly, 310 (81\%) 95\% CI: 79.7-87.3\% thoroughly washed and cleaned fruits/vegetables before eating. Nearly half of pregnant women, 177 (48\%) 95\% CI: 42.7-52.9\%, had been involved in farming or gardening activities before. However, 22.6\% (95\% CI: 18.68-27.2\%) covered their hands with gloves to avoid any possible exposure to disease agents when handling the soil. Table 2 provides more details on the pregnant women's practices towards toxoplasmosis. 
Table 2: The practices that could be linked to T. gondii infection risk among pregnant women at six Reproduction and Child Health clinics in Temeke, Dares Salaam ( $\mathrm{N}=371$ )

\begin{tabular}{|c|c|c|c|c|}
\hline \multirow{2}{*}{ Risk practices and behaviors } & Yes & & No & \\
\hline & $n(\%)$ & $95 \%$, C.I & $n(\%)$ & $95 \%$, C.I \\
\hline Have domestic cat(s) at home & $26(7 \%)$ & $4.3-9.6$ & $345(93 \%)$ & $90.3-95.6$ \\
\hline Involved in changing and cleaning cat litter & $20(5.4 \%)$ & $3.1-7.6$ & $351(94.6 \%)$ & 92.3-96.9 \\
\hline Involved in farming or gardening activities. & $177(48 \%)$ & $42.7-52.9$ & $194(52 \%)$ & $47.0-57.3$ \\
\hline Wear gloves when farming or gardening. & $84(22.6 \%)$ & 18.7-27.2 & $287(77.4 \%)$ & $72.8-81.3$ \\
\hline $\begin{array}{l}\text { Routinely wash hands with soap after } \\
\text { farming/gardening. }\end{array}$ & $133(35.8 \%)$ & $30.9-40.7$ & $238(64.2 \%)$ & $59.2-69.1$ \\
\hline Do eat raw, cured or rare meat. & $38(10.2 \%)$ & 7.1-13.3 & $333(89.8 \%)$ & $86.6-92.8$ \\
\hline Eat raw fruits/vegetables & $69(19 \%)$ & $14.6-22.5$ & $302(81 \%)$ & $77.4-85.3$ \\
\hline $\begin{array}{l}\text { Thoroughly wash and clean fruits or vegetables before } \\
\text { eating. }\end{array}$ & $310(84 \%)$ & 79.7-87.3 & $61(16 \%)$ & $12.6-20.2$ \\
\hline Have received blood donation during pregnancy & $37(10 \%)$ & $6.9-13.0$ & $334(90 \%)$ & $86.9-93.0$ \\
\hline \multicolumn{5}{|l|}{ The source of water } \\
\hline Tap & $250(67 \%)$ & $63.5-72.5$ & $121(33 \%)$ & $14.3-62.6$ \\
\hline Well & $100(27 \%)$ & 22.4-31.4 & $271(73 \%)$ & $71.7-82.8$ \\
\hline Other & $21(6 \%)$ & $3.5-8.1$ & $350(94 \%)$ & 93.1-99.4 \\
\hline Drink untreated water & $214(58 \%)$ & $52.6-63.7$ & $157(42 \%)$ & $37.2-47.3$ \\
\hline
\end{tabular}

\section{The relationship between demographic data and the} practices of pregnant women associated with Toxoplasmosis.

There was a significant statistical relationship between risk prevention practices towards toxoplasmosis and age and the gravidity of pregnant women. Prevention of risk practices towards toxoplasmosis decreased significantly in pregnant women by $78 \%(\mathrm{OR}=0.22,95 \%$ CI: 0.09 $0.59)$ and $74 \%$ (OR $=0.26$, CI: $0.11-0.59)$ for the age of 19-25 and 26-35 years respectively as compared to those $<19$ years. No significant difference was observed for those $>35$ years of age. When adjusted for education, marital status and gestation age, it was found that there were fewer at-risk practices at a young age $(\mathrm{P}<0.01)$ while multigravidae $(\mathrm{OR}=2.65,95 \%$ CI: 1.38-5.08) had significantly greater risk practices towards toxoplasmosis (Table 3).

\section{Awareness of health workers regarding toxoplasmo-} sis

The awareness of health workers of the clinical manifestations of toxoplasmosis in pregnant women and newborn or children were assessed using key ruling out factors. The results are summarized in Table 4. A total of 22 health workers participated in the survey, included were 4 medical officers, 12 nurses, 3 laboratory technicians, 1 sonographer and 2 maternal and child health assistants. Of 22 medical health workers, $36.4 \%$ (95\% CI: $14.5-58.2 \%)$ were aware of toxoplasmosis. The majority of health workers were unaware of the possible transmission of Toxoplasma through sexual intercourse $(77 \%)$ or through consumption of unwashed and raw fruits or vegetables $(86 \%)$. However, all those who were aware of toxoplasmosis agreed that toxoplasmosis was a congenital disease. 
Table 3: Association of risk practices towards toxoplasmosis with the demographic data of 371 pregnant women in six Reproductive and Child Health clinics in Temeke, Dar es Salaam.

\begin{tabular}{llllll}
\hline Factors & & $\begin{array}{l}\text { Crude OR (95\% } \\
\text { C.I) }\end{array}$ & $P$ Value & $\begin{array}{l}\text { Adjusted OR (95\% } \\
\text { C.I) }\end{array}$ & $P$ Value \\
\hline \multirow{2}{*}{ Age groups } & $<19$ & $0.95(0.50-1.77)$ & 0.783 & & \\
& $19-25$ & $0.40(0.19-0.82)$ & 0.013 & $0.26(0.11-0.59)$ & 0.002 \\
& $26-35$ & $0.50(0.24-1.04)$ & 0.064 & $0.22(0.09-0.59)$ & 0.002 \\
& age $>35$ & $1.14(0.49-2.61)$ & 0.762 & $0.48(0.17-1.34)$ & 0.16 \\
Abortion & Yes & $1.28(0.53-3.09)$ & 0.59 & & \\
history & & & & & 0.14 \\
Gravidity & No abortion & $0.61(0.37-0.99)$ & 0.047 & $0.67(0.40-1.13)$ & 0.003 \\
& Multigravidae & $1.94(1.19-3.13)$ & 0.007 & $2.65(1.38-5.08)$ & 0 \\
& Primigravidae & $0.17(0.07-0.41)$ & 0 & & \\
\hline
\end{tabular}

Table 4: Awareness of health workers $(n=22)$ in six Reproductive and Child Health clinics on the clinical manifestations of toxoplasmosis in pregnant women, new-born and children (Temeke municipality, Dar es salaam-Tanzania

\begin{tabular}{|c|c|c|c|}
\hline Clinical manifestation of toxoplasmosis in pregnant women & Yes $\%$ & No $\%$ & $\begin{array}{r}\text { Don’t } \\
\text { know\% }\end{array}$ \\
\hline $\begin{array}{l}\text { Pregnant women can develop serious problems due to } \\
\text { toxoplasmosis }\end{array}$ & $7(32 \%)$ & $1(4 \%)$ & $14(64 \%)$ \\
\hline Toxoplasmosis in pregnant women can cause fever and flu & $6(27 \%)$ & $2(9 \%$ & $14(64 \%)$ \\
\hline $\begin{array}{l}\text { Toxoplasmosis in pregnant women can cause swollen glands } \\
\text { (lymphadenitis) }\end{array}$ & $6(27 \%)$ & $2(9 \%)$ & $14(63.6 \%)$ \\
\hline Toxoplasmosis in pregnant women can be asymptomatic & $5(23 \%)$ & $3(13 \%)$ & $14(63.6 \%)$ \\
\hline
\end{tabular}

\section{Clinical manifestation of toxoplasmosis in children}

Unborn and newborn children can develop serious complications $\quad 8(36 \%) \quad 0(0 \%) \quad 14(63.6 \%)$ due to toxoplasmosis A baby with Toxoplasma infection can have no signs at birth but $7(32 \%) \quad 1(4 \%) \quad 14(63.6 \%)$ develops illness later

$\begin{array}{llll}\text { A baby with Toxoplasmosis can have vision problems } & 8(36 \%) & 0(0 \%) & 14(63.6 \%)\end{array}$

A baby with Toxoplasmosis can have mental problems $\quad 5(23 \%) \quad 3(13 \%) \quad 14(63.6 \%$

\section{Discussion}

The assessment of awareness of congenital toxoplasmosis revealed low level of awareness among health workers and pregnant women attending health care facilities in Temeke municipality, Dar es Salaam, Tanzania. To the best of our knowledge, this was the first study conducted in Tanzania to estimate the awareness level and practices to- wards toxoplasmosis. A few pregnant women and health workers were aware of congenital toxoplasmosis. It is likely that pregnant women may have unknowingly experienced or observed the signs or symptoms of the disease but could not know if toxoplasmosis was responsible for those signs and symptoms. For example, pregnant women said they have had flu-like symptoms and generalized 
or cervical lymphadenitis in their gestation period, but they did not know if toxoplasmosis can also present with the same symptoms.

The low awareness of pregnant women may be attributed to different factors, one being the lack of common indigenous or local name (Swahili) used to describe toxoplasmosis in Tanzania. Low level of education may be another factor. Elsewhere, high level of education has been shown to substantially increase awareness of toxoplasmosis $^{28}$. In our study, most pregnant women had a low education (basic primary education) and all pregnant women who did not attend any school were not aware of toxoplasmosis. It was observed that toxoplasmosis was not one of the diseases of priority for prenatal screening in Tanzania. This, on its own, could have contributed to the low awareness. These findings of low level awareness are in agreement with a study in central Afar region in Ethiopia where low level of knowledge (5.8\%) among pregnant women was recorded 29. Similarly, low knowledge of toxoplasmosis $(11 \%)$ was reported in Malaysia, Philippines and Thailand ${ }^{28}$. Despite the disease burden and the risk of contributing to congenitally transmitted conditions in newborns ${ }^{26}$, maternal and child health care in Tanzania does not consider toxoplasmosis important for prenatal screening, unlike other congenital diseases such as HIV/AIDS, syphilis and malaria.

The consequences of such a policy for children remain uncertain because only seroprevalence studies have been carried out but there has been no research to estimate and determine the incidence of congenital toxoplasmosis or outcomes associated with toxoplasmosis in pregnant women and children or newborns in Tanzania. Granted, the majoriy of women tend to be aware of the diseases for which they are usually screened when they become pregnant. None of the interviewed pregnant women reported having been tested for toxoplasmosis. By implication, absence of prenatal screening services for toxoplasmosis in the country could have contributed to lack of awareness and knowledge of toxoplasmosis. Since the disease is not given any attention in the country's health sector and health workers reported lack of modern tools for routine diagnosis, it was not surprising that most pregnant women and health workers had low awareness of the disease.
While lack of awareness of toxoplasmosis was not associated with the practices of pregnant women towards the disease, most of the pregnant women unknowingly had preventive practices that might limit their exposure to infection by $T$. gondii. They did not eat raw, cured or rare meat, a factor which accounts for most of human Toxoplasma exposure ${ }^{30}$. Similar results were obtained from Malaysia where $12.2 \%$ of the pregnant women ate raw or undercooked meat ${ }^{28}$. In part, preventive practices towards toxoplasmosis among pregnant women in Temeke municipality could also be due to improved sanitation behaviours and standard of living in Temeke. Such practices, however, are not sufficient in themselves for limiting exposure to $T$. gondii. While the majority did not eat raw or undercooked meat, 58\% drank untreated water which could harbour the parasite oocysts and serve as a potential source of Toxoplasma infection.

The current study established a relationship between age and gravidity of pregnant women with risk practices towards Toxoplasma infection. Comparably, there was a greater risk practice of pregnant women older than 19 years towards toxoplasmosis than those below 19. Similarly, risk for $T$. gondii infection also increased with age in previous studies ${ }^{31-35}$. The reasons for the increase of risk of exposure to $T$. gondii by age are still unclear. Like the findings from France and Thailand ${ }^{36,37}$, we found that multigravidae was associated with greater risk for toxoplasmosis based on their practices . Multigravidae women were $93 \%$ at-risk of toxoplasmosis compared to primigravidae. The odds of having clinical toxoplasmosis in multigravidae women can be attributed to age which was found to be a risk factor ${ }^{32-34,36}$.

Like pregnant women, health workers in Temeke municipality reported unexpectedly low levels of awareness towards toxoplasmosis. This is similar to findings in Ethiopia where few health workers were reported to have knowledge of toxoplasmosis ${ }^{29}$. Nurses were the dominant health staff in maternal and child health/reproductive and child health $(\mathrm{MCH} / \mathrm{RCH})$ clinics in Temeke municipality. Despite nurses spending more time with pregnant women than other category of staff, they displayed an extremely low awareness level of congenital toxoplasmosis compared to clinicians and laboratory technicians. This scenario could impede the effectiveness of prenatal care provided in the clinics in Temeke. The 
difference in awareness levels among health workers may be due to professional disciplines and level of education. All health workers who reported awareness of toxoplasmosis studied the disease at some time in medical school. Many of the nurses working in health facilities in Temeke municipality were enrolled nurses who received basic training in nursing. On the other hand, clinicians and laboratory technicians had a tertiary medical education. Due to low staffing of health workers, the only nurse who was aware of the disease practiced both as a nurse and laboratory technician in the $\mathrm{MCH}$ clinic. This finding indicates that performing multiple duties involving crosscutting disciplines may enhance the knowledge of a health worker. In our study, none of the health workers had diagnosed toxoplasmosis in their entire clinical practices. The reasons for not diagnosing the disease were said to be absence of suspected toxoplasmosis cases and lack of diagnostic tools. In addition, lack of routine screening for toxoplasmosis limits the diagnosis of the disease in pregnant women and newborns. With the challenges outlined above, there are chances of over-diagnosis of malaria or unresponsive malaria cases and subsequently under-diagnosis of febrile illness of zoonotic diseases including toxoplasmosis. This is because of similar clinical presentations of both diseases such as fever and a cold.

\section{Limitations}

No serological analysis of interviewed pregnant women was done to complement the questionnaire survey. Only pregnant women and HCWs were interviewed and thus no data for newborns was collected. The study, therefore, did not investigate the risk estimate for the occurrence of congenital toxoplasmosis. Data on clinical presentation in newborns delivered from HIV-negative and HIV-positive women was lacking. Such a gap can be bridged by screening newborns whether serologically, clinically or both in order to get an overview of the importance of this disease in Tanzania.

\section{Conclusions and recommendations}

The current study highlights the low level of awareness and practices towards congenital toxoplasmosis among health workers and pregnant women in Temeke municipality. This low level of awareness may be is due to lack of common indigenous name for toxoplasmosis in Tanzania, low level of education and lack of priority for prenatal toxoplasmosis screening services. Lack of aware- ness is likely to increase the risk of exposure and unprotected practices towards the disease.

As a result of the above, there is a need to upgrade the knowledge on congenital toxoplasmosis in Tanzania. From the findings on HCWs, a question may be asked as to why pregnant women should be aware of a disease which seemingly is not even known to medical personnel in the country. Thus, apart from a push to upgrade knowledge for medical personnel, education of pregnant women is recommended. For the former, this can be done through strengthening of the curriculum for training of enrolled nurses and midwives to cover more aspects of congenitally transmitted diseases including toxoplasmosis. This initiative can be supplemented by the development and provision of prenatal brochures that address different reproductive and child health issues including maternal behaviours' and practices, as well as possible diseases of congenital importance and their risks during pregnancy. However it might be worth investigating, whether screening would be a good approach to enhance the awareness of pregnant women and health workers in regards to congenital toxoplasmosis.

At the government level, an intervention through health systems and policy would support and facilitate screening of toxoplasmosis and other congenitally transmitted diseases among pregnant women attending prenatal health care, as well as in the newborns.

\section{Acknowledgements}

Our sincere thanks go to the University of Zambia particularly School of Veterinary Medicine, the staff at health facilities in Temeke municipality where the study was conducted and pregnant women who consented to participate in this study. We would like to extend our sincere gratitude to Elizabeth Fedrick Ochali and Zubeda Ramadhani Yusuph for their volunteering hearts in assisting data collection.

\section{Competing interests}

The authors declare that they have no competing interests.

\section{References}

1. J. P. Dubey, "Toxoplasmosis - A waterborne zoonosis," Veterinary Parasitology, vol. 126, no. 1-2 SPEC.ISS. Elsevier, pp. 57-72, 09-Dec-2004. 
2. D. J. P. Ferguson et al., "Maternal inheritance and stage-specific variation of the apicoplast in Toxoplasma gondii during development in the intermediate and definitive host," Eukaryot. Cell, vol. 4, no. 4, pp. 814-826, 2005. 3. F. Bertoli, M. Espino, J. R. Arosemena, J. L. Fishback, and J. K. Frenkel, "A spectrum in the pathology of toxoplasmosis in patients with acquired immunodeficiency syndrome.," Arch. Pathol. Lab. Med., vol. 119, no. 3, pp. 214-24, Mar. 1995.

4. M.-L. Dardé, D. Ajzenberg, and C. Su, Molecular Epidemiology and Population Structure of Toxoplasma gondii, Second Edi. Elsevier, 2013.

5. F. Robert-Gangneux and M. L. Dardé, "Epidemiology of and diagnostic strategies for toxoplasmosis," Clin. Microbiol. Rev., vol. 25, no. 2, pp. 264-296, 2012.

6. A. M. Tenter, A. R. Heckeroth, and L. M. Weiss, "Toxoplasma gondii: from animals to humans.," Int. J. Parasitol., vol. 30, no. 12-13, pp. 1217-58, 2000.

7. A. Kijlstra and E. Jongert, "Control of the risk of human toxoplasmosis transmitted by meat," Int. J. Parasitol., vol. 38, no. 12, pp. 1359-1370, 2008.

8. J. G. Montoya and J. S. Remington, "Management of Toxoplasma gondii infection during pregnancy," Clin Infect Dis, vol. 47, no. 4, pp. 554-566, 2008.

9. J. S. Remington, R. McLeod, P. Thulliez, and G. Desmonts, "Toxoplasmosis," in Infectious Diseases of the Fetus and Newborn Infant, Sixth Edit., W.B. Saunders, 2006, pp. 947-1091.

10. J. Flegr, J. Prandota, M. Sovičková, and Z. H. Israili, "Toxoplasmosis - A global threat. Correlation of latent toxoplasmosis with specific disease burden in a set of 88 countries," PLoS One, vol. 9, no. 3, 2014.

11. J. Furtado, J. Smith, R. Belfort, D. Gattey, and K. Winthrop, "Toxoplasmosis: A global threat," J. Glob. Infect. Dis., vol. 3, no. 3, pp. 281-284, 2011.

12. I. Ayi, S. Edu, K. . Apea-kubi, D. Boamah, K. . Bosompem, and D. Edoh, "Sero-epidemiology of toxoplasmosis amongst pregnant women in the greater Accra region of Ghana," Ghana Med.J., vol. 43, no. 3, 2010.

13. J. A. Ohiolei and C. Isaac, "Toxoplasmosis in Nigeria: The story so far (1950-2016): A review," Folia Parasitol. (Praha)., vol. 63, no. 030, pp. 1803-646, 2016.

14. M. De Paschale et al., "Antenatal screening for Toxoplasma gondii, Cytomegalovirus, rubella and Treponema pallidum infections in northern Benin," Trop. Med. Int. Heal., vol. 19, no. 6, pp. 743-746, 2014.

15. W. Gelaye, T. Kebede, and A. Hailu, "High prevalence of anti-toxoplasma antibodies and absence of Toxoplasma gondii infection risk factors among pregnant women attending routine antenatal care in two Hospitals of Addis Ababa, Ethiopia," Int. J. Infect. Dis., vol. 34, no. 2015, pp. 41-5, 2015.

16. B. Lelong et al., "Prevalence of toxoplasmosis in a population of pregnant women in Antananarivo (Madagascar)," Bull. Soc. Pathol. Exot., vol. 88, no. 1, pp. 46-49, 1995.

17. I. Lindström, D. H. Kaddu-Mulindwa, F. Kironde, and J. Lindh, "Prevalence of latent and reactivated Toxoplasma gondii parasites in HIV-patients from Uganda," Acta Trop., vol. 100, no. 3, pp. 218-222, 2006.

18. C. Frimpong, M. Makasa, L. Sitali, and C. Michelo, "Seroprevalence and determinants of toxoplasmosis in pregnant women attending antenatal clinic at the university teaching hospital, Lusaka, Zambia," BMC Infect. Dis., vol. 17, no. 1, 2017.

19. K. Kistiah, A. Barragan, J. Winiecka-Krusnell, A. Karstaedt, and J. Frean, "Seroprevalence of Toxoplasma gondii infection in HIV-positive and HIV-negative subjects in Gauteng, South Africa," South. African J. Infect. Dis., vol. 26, no. 4, pp. 225-228, 2011.

20. C. W. Liao et al., "Seroprevalence of Toxoplasma gondii infection among children in Swaziland, southern Africa," Ann. Trop. Med. Parasitol., vol. 103, no. 8, pp. 731-736, Dec. 2013.

21. Y. Doudou et al., "Toxoplasmosis among pregnant women: High seroprevalence and risk factors in Kinshasa, Democratic Republic of Congo," Asian Pac. J. Trop. Biomed., vol. 4, no. 1, pp. 69-74, 2014.

22. A. Bouratbine, E. Siala, M. K. Chahed, K. Aoun, and R. Ben Ismail, "Sero-epidemiologic profile of toxoplasmosis in northern Tunisia," Parasite, vol. 8, no. 1, pp. 6166, 2001.

23. E. Gille, A. Björkman, I. Rooth, I. Ljungstrom, and E. Linder, "Low seroprevalence of Toxoplasma gondii antibodies in a Tanzanian village," Trans. R. Soc. Trop. Med. Hyg., vol. 86, no. 3, pp. 263-5, 1992.

24. E. R. Shao et al., "Sero-Prevalence and Factors Associated with Toxoplasma gondii Infection among Pregnant Women Attending Antenatal Care in the Referral Hospital in Tanzania: Cross Sectional Study," vol. 3, no. 2, 2015. 25. B. Mwambe et al., "Sero-prevalence and factors associated with Toxoplasma gondii infection among pregnant women attending antenatal care in Mwanza, Tanzania," Parasit. Vectors, vol. 6, no. 1, p. 222, 2013. 
26. E. Swai and L. Schoonman, "Seroprevalence of Toxoplasma gondii infection amongst residents of Tanga District in north-east Tanzania," Tanzan. J. Health Res., vol. 11, no. 4, pp. 205-9, Jan. 2010.

27. S. Lwanga and S. Lemeshow, "Sample size determination in health studies: A practical manual.," World Heal. Organ. Geneva, vol. 88, pp. 1-22, 1991.

28. H. Andiappan et al., "Knowledge and practice on Toxoplasma infection in pregnant women from Malaysia, Philippines, and Thailand," Front. Microbiol., vol. 5, p. 291, 2014.

29. A. Hadush Desta, "Knowledge, Attitude and Practice of Community Towards Zoonotic Importance of Toxoplasma Infection in Central Afar Region, North East Ethiopia," Int. J. Biomed. Sci. Eng., vol. 3, no. 6, pp. 74-81, 2015.

30. J. Montoya and O. Liesenfeld, "Toxoplasmosis," Lancet, vol. 363, no. 9425, pp. 1965-76, 2004.

31. J. L. Jones, "Toxoplasma gondii Infection in the United States: Seroprevalence and Risk Factors," Am. J. Epidemiol., vol. 154, no. 4, pp. 357-365, 2001.

32. O. E. Sousa, R. E. Saenz, and J. K. Frenkel, "Toxoplasmosis in Panama: A 10-year study," Am. J. Trop. Med. Hyg., vol. 38, no. 2, pp. 315-22, 1988.
33. M. Tourdjman, C. Tcheandjieu, H. de Valk, V. Goulet, and Y. le Strat, "Toxoplasmosis among pregnant women in France: trends in seroprevalence and associated factors between 1995 and 2010.," Depistages au cours la grossesse a la Naiss. donnees Epidemiol. Recent., vol. 15, no. 16, pp. 264-272, 2015.

34. H. I. AL Mohammad, T. T. Amin, M. H. Balaha, and M. S. AL Moghannum, "Toxoplasmosis among the pregnant women attending a Saudi maternity hospital: seroprevalence and possible risk factors," Ann. Trop. Med. Parasitol., vol. 104, no. 6, pp. 493-504, 2010.

35. J. B. McAuley, “Congenital Toxoplasmosis.," J. Pediatric Infect. Dis. Soc., vol. 3, no. 1, pp. 30-5, Sep. 2014.

36. F. Berger, V. Goulet, Y. Le Strat, and J. C. Desenclos, "Toxoplasmosis among pregnant women in France: Risk factors and change of prevalence between 1995 and 2003," Rev. Epidemiol. Sante Publique, vol. 57, no. 4, pp. 241-248, 2009.

37. V. Nissapatorn, C. Suwanrath, N. Sawangjaroen, L. Y. Ling, and V. Chandeying, "Toxoplasmosis-serological evidence and associated risk factors among pregnant women in southern Thailand," Am. J. Trop. Med. Hyg., vol. 85, no. 2, pp. 243-247, 2011. 